PROCEEDINGS OF THE

AMERICAN MATHEMATICAL SOCIETY

Volume 139, Number 4, April 2011, Pages 1187-1200

S 0002-9939(2010)10728-1

Article electronically published on November 2, 2010

\title{
A NEGATIVE ANSWER TO A QUESTION OF BASS
}

\author{
G. CORTIÑAS, C. HAESEMEYER, MARK E. WALKER, AND C. WEIBEL \\ (Communicated by Irena Peeva)
}

\begin{abstract}
We address Bass' question, on whether $K_{n}(R)=K_{n}(R[t])$ implies $K_{n}(R)=K_{n}\left(R\left[t_{1}, t_{2}\right]\right)$. In a companion paper, we establish a positive answer to this question when $R$ is of finite type over a field of infinite transcendence degree over the rationals. Here we provide an example of an isolated surface singularity over a number field for which the answer the Bass' question is "no" when $n=0$.
\end{abstract}

\section{INTRODUCTION}

In 1972, H. Bass posed the following question (see 2, question $(\mathrm{VI})_{n}$ ):

Does $K_{n}(R)=K_{n}(R[t])$ imply that $K_{n}(R)=K_{n}\left(R\left[t_{1}, t_{2}\right]\right)$ ?

Bass' question was inspired by Traverso's theorem [20, from which it follows that $\operatorname{Pic}(R)=\operatorname{Pic}(R[t])$ implies $\operatorname{Pic}(R)=\operatorname{Pic}\left(R\left[t_{1}, t_{2}\right]\right)$.

In our companion paper [5], we show that the answer to Bass' question is "yes" for rings of finite type over fields having infinite transcendence degree over $\mathbb{Q}$. In this paper, we give an example showing the answer is "no" in general, even when $n=0$. That is, there is a ring $R$ for which every finitely generated projective module over $R[t]$ is the extension, up to stable isomorphism, of a projective module over $R$, but not every finitely generated projective module over $R\left[t_{1}, t_{2}\right]$ is so extended.

Our example is the isolated hypersurface singularity

$$
R=F[x, y, z] /\left(z^{2}+y^{3}+x^{10}+x^{7} y\right),
$$

where $F$ is any algebraic field extension of $\mathbb{Q}$. (The proof is given in Theorem 4.1) This example was first studied by J. Wahl [21.

Our proof that $R$ indeed gives a negative answer to Bass' question uses what we call generalized $d u$ Bois invariants, $b^{p, q}$, of an isolated singularity in characteristic zero; see (2.8). The (ordinary) du Bois invariants were introduced by Steenbrink [18] using the du Bois complexes $\underline{\Omega}^{p}, p \geq 0$. They can equivalently be defined using

Received by the editors April 18, 2010.

2010 Mathematics Subject Classification. Primary 19A49, 19D50; Secondary 19D55, 14F20.

The first author's research was supported by CONICET and partially supported by grants PICT 2006-00836, UBACyT X051, PIP 112-200801-00900, and MTM2007-64704 (Feder funds).

The second author's research was partially supported by NSF grant DMS-0652860.

The third author's research was partially supported by NSF grant DMS-0601666.

The fourth author's research was supported by NSA grant MSPF-04G-184 and the Oswald Veblen Fund.

(C)2010 American Mathematical Society Reverts to public domain 28 years from publication 
sheaf cohomology in Voevodsky's $c d h$ topology thanks to the natural isomorphism (see Lemma 2.1)

$$
\mathbb{H}_{\text {zar }}^{*}\left(X, \underline{\Omega}_{X}^{p}\right) \cong H_{\text {cdh }}^{*}\left(X, \Omega^{p}\right) .
$$

The generalized du Bois invariants are defined as the cohomology of the complex obtained by patching together the du Bois complexes $\underline{\Omega}^{p}$ and the higher cotangent complexes used to define André-Quillen homology. The Euler characteristics of these patched together complexes, written $\chi^{p}$ for $p \geq 0$, turn out to be constant in suitably nice families (see Theorem 2.14). In particular, we prove in Proposition 4.3 that $\chi^{p}\left(R_{a}\right)$ is independent of $a \in F$, where $R_{a}=F[x, y, z] /\left(z^{2}+y^{3}+x^{10}+a x^{7} y\right)$. Since the ring $R_{0}$ is graded, the values of $\chi^{p}\left(R_{0}\right)=\chi^{p}\left(R_{1}\right)$ are easy to compute, and these computations allow us to prove our assertion about $R=R_{1}$.

Notation. Throughout this paper, $F$ denotes a field of characteristic zero. By "a scheme over $F$ " we mean a separated scheme of finite type over $F$. We write Sch/F for the category of all such schemes. Unless otherwise stated, Hochschild homology and modules of Kähler differentials will be taken relative to $F$. That is, we write $\Omega_{X}^{q}$ and $H H_{q}(X)$ for $\Omega_{X / F}^{q}$ and $H H_{q}(X / F)$.

\section{On $c d h$-COHOMOLOGY AND NIL $K$-THEORY}

For any functor $G$ from rings to an abelian category, $N G$ is the functor with $N G(R)$ defined to be the kernel of the map $G(R[t]) \rightarrow G(R)$ induced by evaluation at $t=0$. Since $G(R[t]) \rightarrow G(R)$ is split by the canonical map $G(R) \rightarrow G(R[t])$, the functor $N G$ is a summand of the functor $R \mapsto G(R[t])$. We define $N^{2} G=N(N G)$.

It is convenient to phrase Bass' question in terms of Bass' Nil groups, $N K_{*}(R)$, as follows:

$$
\text { Does } N K_{n}(R)=0 \text { imply that } N^{2} K_{n}(R)=0 \text { ? }
$$

Our example uses the following theorem from our companion paper [5]. (The notation in this theorem is discussed below; the particular forms of $V$ and $W$ in this theorem reflect extra structure not relevant for this paper.)

Theorem 1.1 ([5, Theorems 0.1 and 0.7]). Let $R$ be a normal domain of dimension 2 that is of finite type over $\mathbb{Q}$. Then, letting $V$ and $W$ denote the countably infinitedimensional $\mathbb{Q}$ vector spaces $t \mathbb{Q}[t]$ and $\Omega_{\mathbb{Q}[t]}^{1}$, we have:

a) $N K_{0}(R) \cong H_{\text {cdh }}^{1}\left(R, \Omega^{1}\right) \otimes_{\mathbb{Q}} V$.

b) $N K_{-1}(R) \cong H_{\text {cdh }}^{1}(R, \mathcal{O}) \otimes_{\mathbb{Q}} V$.

c) If $N K_{0}(R)=0$, then $K_{0}\left(R\left[t_{1}, t_{2}\right]\right) \cong K_{0}(R) \oplus\left(N K_{-1}(R) \otimes_{\mathbb{Q}} W\right)$.

In particular, for $R$ as in Theorem 1.1 the answer to Bass' question with $n=0$ is "no" if and only if $H_{\text {cdh }}^{1}\left(R, \Omega^{1}\right)=0$ and $H_{\text {cdh }}^{1}(R, \mathcal{O}) \neq 0$.

Recall that the $c d h$ topology on $\mathrm{Sch} / F$, written $(\mathrm{Sch} / F)_{\mathrm{cdh}}$, is the Grothendieck topology generated by Nisnevich open covers and abstract blow-up squares [19. If $\mathcal{G}$ is a presheaf on $\mathrm{Sch} / F$, by $H_{\mathrm{cdh}}^{*}(X, \mathcal{G})$, we mean the $c d h$-sheaf cohomology of the $c d h$-sheafification $\mathcal{G}$. For example, $H_{\mathrm{cdh}}^{*}\left(X, \Omega^{p}\right)$, for $p \geq 0$, refers to the $c d h$ cohomology of the $c d h$-sheafification of $Y \mapsto \Omega_{Y}^{p}$. (Of course, $\Omega_{Y}^{0}=\mathcal{O}_{Y}$.) When $X=\operatorname{Spec} R$ for an $F$-algebra $R$ of finite type over $F$, we usually write $H_{\text {cdh }}^{*}(R, \mathcal{G})$ for $H_{\mathrm{cdh}}^{*}(\operatorname{Spec} R, \mathcal{G})$. 


\section{Generalized du Bois invariants, $\chi^{p}$ And Deformations}

In this section, we construct invariants of isolated singularities, called the generalized du Bois invariants $b^{p, q} \in \mathbb{N}$, which for $q>0$ coincide with the du Bois invariants introduced by Steenbrink [18. For isolated singularities that are also local complete intersections, for each fixed $p$ only a finite number of the integers $b^{p, q}$ are nonzero. Thus it makes sense to define $\chi^{p}:=\sum_{q}(-1)^{q} b^{p, q}$ in this situation. The main result of this section is Theorem 2.14, that the $\chi^{p}$ are invariant under suitably nice deformations.

Recall that we work over a field $F$ of characteristic zero. Several of the results we quote from here on, in particular, anything involving du Bois complexes have been proved under the assumption that $F=\mathbb{C}$. However, flat base change implies that they all remain valid over an arbitrary field $F$ of characteristic 0 .

Fix a scheme $X$ of finite type over $F$ and choose a proper simplicial hyperresolution $\pi: Y_{\bullet} \rightarrow X$. Following [7] we fix $p$ and we consider the $p$-th $d u$ Bois complex

$$
\underline{\Omega}_{X}^{p}=\mathbb{R} \pi_{*} \Omega_{Y_{\bullet}}^{p}
$$

Du Bois shows in [7] that the assignment $X \mapsto \underline{\Omega}_{X}^{p}$ is natural in $X$ up to unique isomorphism in the derived category. The relevance for us lies in the fact that the Zariski hypercohomology of the complex $\underline{\Omega}_{X}^{p}$ computes $H_{\mathrm{cdh}}^{*}\left(X, \Omega^{p}\right)$ :

Lemma 2.1. Let $X$ be a scheme of finite type over $F$ and $p \geq 0$. Then there is a natural isomorphism

$$
\mathbb{H}_{\mathrm{zar}}^{*}\left(X, \underline{\Omega}_{X}^{p}\right) \cong H_{\mathrm{cdh}}^{*}\left(X, \Omega^{p}\right) .
$$

A very similar observation for the $h$-topology has been made by Ben Lee [13]; the proof we give here is based upon the proof of [4, 4.1].

Proof. Recall that $H_{\mathrm{cdh}}^{*}\left(X, \Omega^{p}\right)$ is the Zariski hypercohomology of the complex $\left.\mathbb{R} a_{*} a^{*} \Omega^{p}\right|_{X}$, where $a:(\mathrm{Sch} / F)_{\text {cdh }} \rightarrow(\mathrm{Sch} / F)_{\text {zar }}$ is the morphism of sites and $\left.\right|_{X}$ denotes the restriction from the big Zariski site $(\mathrm{Sch} / F)_{\mathrm{zar}}$ to $X_{\text {zar }}$. Let $Y_{\bullet} \rightarrow X$ be a simplicial hyperresolution. By [6, 2.5], we have a quasi-isomorphism on $X_{\text {zar }}$,

$$
\left.\Omega_{Y_{n}}^{p} \stackrel{\simeq}{\longrightarrow} \mathbb{R} a_{*} a^{*} \Omega^{p}\right|_{Y_{n}},
$$

since each $Y_{n}$ is smooth. Using also [4, 4.3], we have a diagram of equivalences

$$
\left.\mathbb{R} a_{*} a^{*} \Omega^{p}\right|_{X} \stackrel{\simeq}{\longrightarrow} \mathbb{R} \pi_{*}\left(\left.\mathbb{R} a_{*} a^{*} \Omega^{p}\right|_{Y_{\bullet}}\right) \stackrel{\simeq}{\simeq} \mathbb{R} \pi_{*} \Omega_{Y_{\bullet}}^{p}=\underline{\Omega}_{X}^{p} .
$$

Applying $\mathbb{H}_{\text {zar }}^{*}(X,-)$ yields $H_{\text {cdh }}^{*}\left(X, \Omega^{p}\right) \cong \mathbb{H}_{\text {zar }}^{*}\left(X, \underline{\Omega}^{p}\right)$.

Isolated singularities. Suppose that $\operatorname{Sing}(X)$ is an isolated point $x$. Choose a good resolution $\pi: Y \rightarrow X$, meaning that $Y$ is smooth, $\pi$ is proper and an isomorphism away from $x$, and $E=\pi^{-1}(x)_{\text {red }}$ is a normal crossings divisor with smooth components. Then by [7, 4.8, 4.11] we have a distinguished triangle

$$
0 \rightarrow \underline{\Omega}_{X}^{p} \rightarrow \mathbb{R} \pi_{*} \Omega_{Y}^{p} \oplus \Omega_{x}^{p} \rightarrow \mathbb{R} \pi_{*} \underline{\Omega}_{E}^{p} \rightarrow 0 .
$$

To rewrite this, let $E_{1}, \ldots, E_{t}$ denote the (smooth) components of $E$, and define

$$
Y_{n}= \begin{cases}Y \amalg x_{0} & n=0, \\ \coprod_{i_{1}<\cdots<i_{n}} E_{i_{1}} \times_{Y} \cdots \times_{Y} E_{i_{n}} & n>0 .\end{cases}
$$

By [7, 4.10], the complex $\underline{\Omega}_{E}^{p}$ is quasi-isomorphic to (the total complex of)

$$
\underline{\Omega}_{Y_{1}}^{p} \rightarrow \underline{\Omega}_{Y_{2}}^{p} \rightarrow \cdots
$$


The maps in this complex are given by the usual alternating sum of restriction maps, since the complex arises from a coskeletal hyperresolution of $E$. Generically writing $\pi: Y_{n} \rightarrow X$ for the canonical map from $Y_{n}$ to $X$, we have

$$
\underline{\Omega}_{X}^{p} \simeq \operatorname{Tot}\left(\mathbb{R} \pi_{*} \underline{\Omega}_{Y_{0}}^{p} \rightarrow \mathbb{R} \pi_{*} \underline{\Omega}_{Y_{1}}^{p} \rightarrow \mathbb{R} \pi_{*} \underline{\Omega}_{Y_{2}}^{p} \rightarrow \cdots\right) .
$$

Now suppose that $\operatorname{dim}(X)=2$. Because $E_{i} \times_{Y} E_{j} \times_{Y} E_{l}=\emptyset$ for distinct $i, j, l$ and $\Omega_{E_{i} \times_{Y} E_{j}}^{p}=0$ for $i \neq j$ and $p>0$, (2.3) reduces to $\underline{\Omega}_{X}^{p} \simeq \operatorname{Tot}\left(\mathbb{R} \pi_{*} \Omega_{Y}^{p} \rightarrow \bigoplus_{i} \mathbb{R} \pi_{*} \Omega_{E_{i}}^{p}\right)$ for $p>0$, and

$$
\underline{\Omega}_{X}^{0} \simeq \operatorname{Tot}\left(\mathbb{R} \pi_{*} \mathcal{O}_{Y} \oplus \mathcal{O}_{x} \rightarrow \bigoplus_{i} \mathbb{R} \pi_{*} \mathcal{O}_{E_{i}} \rightarrow \bigoplus_{i<j} \mathbb{R} \pi_{*} \mathcal{O}_{E_{i} \times Y} E_{j}\right) .
$$

In other words, in the notation of [21],

$$
\underline{\Omega}_{X}^{0} \simeq \mathbb{R} \pi_{*} \mathcal{O}_{Y}(-E) \oplus \mathcal{O}_{x} \quad \text { and } \quad \underline{\Omega}_{X}^{p} \simeq \mathbb{R} \pi_{*}\left(\Omega_{Y}^{p}(\log E)(-E)\right), \quad p>0 .
$$

Du Bois invariants. Suppose for simplicity that $X=\operatorname{Spec} R$, where $R$ is a domain of finite type over $F$. For any $p \geq 0$, there is a map from the $p$-th higher cotangent complex $\mathcal{L}_{X}^{p}$ (see [14, 3.5.4]) to the $p$-th du Bois complex $\underline{\Omega}_{X}^{p}$, obtained by composing the isomorphism $H_{0}\left(\mathcal{L}_{X}^{p}\right) \cong \Omega_{X}^{p}$ and the natural map $\Omega_{X}^{p} \rightarrow H^{0}\left(\underline{\Omega}_{X}^{p}\right)$.

Definition 2.5. Define the cochain complex $C_{X}^{p}$ of quasi-coherent $\mathcal{O}_{X}$-modules by

$$
C_{X}^{p}:=\operatorname{cone}\left(\mathcal{L}_{X}^{p} \rightarrow \underline{\Omega}_{X}^{p}\right)
$$

That is, we have a triangle $\mathcal{L}_{X}^{p} \rightarrow \underline{\Omega}_{X}^{p} \rightarrow C_{X}^{p} \rightarrow \mathcal{L}_{X}^{p}[1]$.

In the language of [5], the complex $C_{X}^{p}$ gives the homotopy fiber $\mathcal{F}_{H H}$ of the map from the Hochschild complex of $X$ to its $c d h$-fibrant replacement:

$$
\mathbb{H}^{i}\left(C_{X}^{p}\right)=H^{i+1-p}\left(\mathcal{F}_{H H}^{(p)}(X)\right) .
$$

Note that the hypercohomology sheaves of $C_{X}^{p}$ are coherent because the Kähler differentials are taken over $F$. Using Lemma 2.1, 14, 4.5.13] and [5, Lemma 3.4], we conclude that

$$
\mathbb{H}^{q}\left(C_{X}^{p}\right)= \begin{cases}\mathbb{H}^{q}\left(X, \underline{\Omega}^{p}\right) & \text { for } q \geq 1, \\ \operatorname{coker}\left(\Omega_{X}^{p} \rightarrow \mathbb{H}^{0}\left(X, \underline{\Omega}^{p}\right)\right) & \text { for } q=0, \\ \operatorname{ker}\left(\Omega_{X}^{p} \rightarrow \mathbb{H}^{0}\left(X, \underline{\Omega}^{p}\right)\right) & \text { for } q=-1, \\ D_{-1-q}^{(p)}(X) & \text { for } q \leq-2,\end{cases}
$$

where $D_{n}^{(p)}$ denotes André-Quillen homology. Recall that $D_{n}^{(p)}(R) \cong H H_{p+n}^{(p)}(R)$, where

$$
H H_{*}=\prod_{p \geq 0} H H_{*}^{(p)}
$$

is the Hodge decomposition of Hochschild homology.

If $X$ has isolated singularities, then each of the hypercohomology modules $\mathbb{H}^{n}\left(C_{X}^{p}\right)$ is of finite length. In this case we may define, following and expanding on Steenbrink's definition [18, the generalized du Bois invariants to be the numbers

$$
b^{p, q}=b_{X}^{p, q}=\text { length } \mathbb{H}^{q}\left(C_{X}^{p}\right), \quad \text { for } p \geq 0 \text { and } q \in \mathbb{Z} .
$$

Example 2.8.1. For $p=0$, we have $b^{0, q}=0$ if $q<0$. When $R$ is a domain, $b^{0,0}=\operatorname{length}_{R}\left(R^{+} / R\right)$, where $R^{+}$is the seminormalization of $R$, because $\mathcal{L}^{0}=\mathcal{O}_{X}$ and $H_{\mathrm{cdh}}^{0}(R, \mathcal{O})=R^{+}$by [5, 2.5]. If $q>0$, then (2.4) yields $b^{0, q}=h^{q}\left(\mathcal{O}_{Y}(-E)\right)$. 
If, moreover, $X$ is locally a complete intersection, then $H H_{n}^{(p)}(R)=0$ for $n \gg 0$ (see [8]); hence it follows from (2.7) that $C_{X}^{p}$ is homologically bounded.

Definition 2.9. For a local complete intersection $X \in \mathrm{Sch} / F$ with only isolated singularities, define $\chi^{p}(X)$ for $p \geq 0$ to be the Euler characteristic of $C_{X}^{p}$ :

$$
\chi^{p}(X):=\sum_{q}(-1)^{q} b_{X}^{p, q} .
$$

Lemma 2.10. If $X=\operatorname{Spec}(R)$ for a ring $R$ that admits a nonnegative grading with $R_{0}=k$, then $\sum(-1)^{p} b_{X}^{p, q}=0$ for all $q$.

Proof. The cases $q=-1, q=0, q>0$ follow from (2.7) using the exact sequences

$$
\begin{aligned}
0 & \rightarrow \operatorname{nil}(R) \rightarrow \operatorname{tors} \Omega_{R}^{1} \rightarrow \operatorname{tors} \Omega_{R}^{2} \rightarrow \operatorname{tors} \Omega_{R}^{3} \rightarrow \cdots, \\
0 & \rightarrow\left(R^{+} / R\right) \rightarrow \Omega_{\mathrm{cdh}}^{1}(R) / \Omega_{R}^{1} \rightarrow \Omega_{\mathrm{cdh}}^{2}(R) / \Omega_{R}^{2} \rightarrow \cdots, \\
0 & \rightarrow H_{\mathrm{cdh}}^{n}(R, \mathcal{O}) \stackrel{d}{\longrightarrow} H_{\mathrm{cdh}}^{n}\left(R, \Omega^{1}\right) \stackrel{d}{\longrightarrow} H_{\mathrm{cdh}}^{n}\left(R, \Omega^{2}\right) \rightarrow \cdots, \quad n>0,
\end{aligned}
$$

respectively, which are established in [5, Example 3.9]. For $q<-1$ the formula follows from Goodwillie's Theorem [22, 9.9.1].

A key property of $\chi^{p}$ is its invariance under deformations of the sort described in the following theorem. In it, we write $X_{s}$ for the fiber of $X$ over a point $s \in S$.

Theorem 2.14. Suppose $X \rightarrow S$ is a flat local complete intersection map of affine varieties with $S$ smooth and such that the singular locus $X_{\operatorname{sing}}$ of $X$ is finite and étale over $S$. Suppose in addition that one can find a projective map $\pi: Y \rightarrow X$ which is an isomorphism away from $X_{\text {sing }}$, such that $Y$ is smooth and such that the reduced, irreducible components $E_{1}, \ldots, E_{m}$ of $Y \times_{X} X_{\operatorname{sing}}$ are smooth over $S$ and satisfy the property that each

$$
E_{i_{1}, \ldots, i_{t}}:=E_{i_{1}} \times_{Y} E_{i_{2}} \times_{Y} \cdots \times_{Y} E_{i_{t}} \rightarrow S
$$

is smooth $\left(1 \leq i_{1}, \ldots, i_{t} \leq m\right)$. Then $\chi^{p}\left(X_{s}\right)$ is independent of the closed point $s$.

Suppose in addition that a finite group $G$ acts on both $X$ and $Y$ and that $\pi$ and $X \rightarrow S$ are equivariant, where we declare the action of $G$ on $S$ to be trivial. Assume that $X / G \rightarrow S$ is a flat local complete intersection such that $(X / G)_{\operatorname{sing}}$ is finite and étale over $S$. Then $\chi^{p}\left(X_{s} / G\right)$ is independent of the closed point $s \in S$.

Proof. In analogy with Definition 2.5, we use (2.3) to define a relative version of $C^{p}$ :

$$
C_{X / S}^{p}:=\operatorname{Tot}\left(\mathcal{L}_{X / S}^{p} \rightarrow \mathbb{R} \pi_{*} \underline{\Omega}_{Y_{0} / S}^{p} \rightarrow \mathbb{R} \pi_{*} \underline{\Omega}_{Y_{1} / S}^{p} \rightarrow \mathbb{R} \pi_{*} \underline{\Omega}_{Y_{2} / S}^{p} \rightarrow \cdots\right),
$$

where, as in (2.2),

$$
Y_{n}= \begin{cases}Y \amalg X_{\text {sing }} & n=0, \\ \amalg_{i_{1}<\cdots<i_{n}} E_{i_{1}, \ldots, i_{n}} & n>0,\end{cases}
$$

and $\mathcal{L}_{X / S}^{p}$ is the $p$-th cotangent complex for $X \rightarrow S$; the map $\mathcal{L}_{X / S}^{p} \rightarrow \mathbb{R} \pi_{*} \Omega_{Y_{0} / S}^{p}$ is induced by the composite of the natural maps $\mathcal{L}_{X / S}^{p} \rightarrow \Omega_{X / S}^{p} \rightarrow \pi_{*} \Omega_{Y_{0} / S}^{p}$.

The complex $C_{X / S}^{p}$ is a complex of quasi-coherent $\mathcal{O}_{X}$-modules with only finitely many nonzero homology sheaves, each of which is coherent. Moreover, each such homology sheaf is supported on the singular locus of $X$, which maps finitely to $S$. By restriction of scalars along the affine map $X \rightarrow S$, we may therefore regard 
$C_{X / S}^{p}$ as a complex of quasi-coherent $\mathcal{O}_{S}$-modules whose homology is coherent. As such, this complex determines a class $\left[C_{X / S}^{p}\right]$ in $G_{0}(S)=K_{0}(S)$. Explicitly, this class is the alternating sum of these homology modules.

For any point $s \in S$, let $j_{s}: s \rightarrow S$ be the induced map of schemes and let $j_{s}^{*}: K_{0}(S) \rightarrow K_{0}(s) \cong \mathbb{Z}$ be the pull-back map in $K$-theory. Note that for any $s$, the map $j_{s}^{*}$ sends the class of a locally free $\mathcal{O}_{S}$-module to its rank. Consequently, the map $j_{s}^{*}: K_{0}(S) \rightarrow \mathbb{Z}$ does not depend on the choice of $s \in S$. We now prove that for any closed point $s \in S$ :

$$
j_{s}^{*}\left[C_{X / S}^{p}\right]=\left[C_{X_{s} / s}^{p}\right] .
$$

Since the class $\left[C_{X_{s} / s}^{p}\right]$ in $K_{0}(s)=\mathbb{Z}$ is $\chi^{p}\left(X_{s}\right)$ when $s \in S$ is a closed point, this will prove the first assertion of the theorem.

Note first of all that if $\mathcal{F}^{\bullet}$ is any complex of quasi-coherent $\mathcal{O}_{S}$-modules with bounded, coherent homology, then $j_{s}^{*}\left[\mathcal{F}^{\bullet}\right]=\left[\mathbb{L} j_{s}^{*} \mathcal{F}^{\bullet}\right]$, where $\mathbb{L} j_{s}^{*}$ denotes the left derived functor associated to $j_{s}^{*}$. For any $n$, let $\tilde{\pi}: Y_{n} \rightarrow S$ be the structure map, which we are supposing to be smooth and hence flat. Thus $\tilde{\pi}$ and $j_{s}$ are Tor-independent over $S$. Consider the pullback diagram

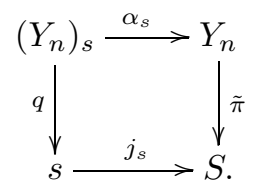

By [1, IV.3.1], we have $\mathbb{L} j_{s}^{*} \mathbb{R} \tilde{\pi}_{*} \Omega_{Y_{n} / S}^{p} \simeq \mathbb{R} q_{*} \mathbb{L} \alpha_{s}^{*} \Omega_{Y_{n} / S}^{p}$. Since $Y_{n} / S$ is smooth, $\Omega_{Y_{n} / S}^{p}$ is locally free and we have

$$
\mathbb{L} \alpha_{s}^{*} \Omega_{Y_{n} / S}^{p}=\alpha_{s}^{*} \Omega_{Y_{n} / S}^{p} \cong \Omega_{\left(Y_{n}\right)_{s} / s}^{p} .
$$

Hence

$$
\mathbb{L} j_{s}^{*} \mathbb{R} \tilde{\pi}_{*} \Omega_{Y_{n} / S}^{p} \simeq \mathbb{R} q_{*} \Omega_{\left(Y_{n}\right)_{s} / s}^{p} .
$$

Similarly, it is a standard property of the cotangent complex that

$$
j_{s}^{*} \mathcal{L}_{X / S}^{p} \simeq \mathbb{L} j_{s}^{*} \mathcal{L}_{X / S}^{p} \simeq \mathcal{L}_{X_{s} / s}^{p} .
$$

Combining these, we get the formula

$$
j_{s}^{*}\left[C_{X / S}^{p}\right]=\left[\cdots \rightarrow \mathcal{L}_{X_{s} / s}^{p} \rightarrow \mathbb{R} q_{*} \Omega_{\left(Y_{0}\right)_{s} / s}^{p} \rightarrow \mathbb{R} q_{*} \Omega_{\left(Y_{1}\right)_{s} / s}^{p} \rightarrow \cdots\right] .
$$

Finally, if $s$ is a closed point, then by (2.3) we have

$$
\underline{\Omega}_{X_{s}}^{p} \simeq\left(\mathbb{R} q_{*} \Omega_{\left(Y_{0}\right)_{s} / s}^{p} \rightarrow \mathbb{R} q_{*} \Omega_{\left(Y_{1}\right)_{s} / s}^{p} \rightarrow \cdots\right)
$$

and hence the formula $j_{s}^{*}\left[C_{X / S}^{p}\right]=\left[C_{X_{s} / s}^{p}\right]$ of (2.14b) , proving the first assertion.

Suppose now that a finite group $G$ acts on $X$ and $Y$ as in the statement of the theorem. Let $Y_{n}$ be as in (2.14a) above; then $G$ acts on $Y_{n} \rightarrow S$ and hence on $\underline{\Omega}_{Y_{n} / S}^{p}$ and $\mathbb{R} \tilde{\pi}_{*} \underline{\Omega}_{Y_{n} / S}^{p}$ for all $n$. For each $s \in S$, the group $G$ acts also on $\underline{\Omega}_{\left(Y_{n}\right)_{s}}^{p}$.

Since $G$ is a finite group and we are in characteristic 0 , taking $G$-invariants is exact. This implies the key property we will need, proven in [7, 5.12], namely that

$$
\underline{\Omega}_{\left(Y_{n}\right)_{s} / G}^{p} \simeq\left(\underline{\Omega}_{\left(Y_{n}\right)_{s}}^{p}\right)^{G} \simeq\left(\Omega_{\left(Y_{n}\right)_{s}}^{p}\right)^{G} .
$$


Since taking $G$-invariants also commutes with $\mathbb{R} q_{*}$, this property implies that

$$
\mathbb{R} q_{*}\left(\underline{\Omega}_{\left(Y_{n}\right)_{s} / G}^{p}\right) \simeq \mathbb{R} q_{*}\left(\left(\Omega_{\left(Y_{n}\right)_{s}}^{p}\right)^{G}\right) \simeq\left(\mathbb{R} q_{*} \Omega_{\left(Y_{n}\right)_{s}}^{p}\right)^{G} .
$$

Define the analogue $D_{X / S}^{p}$ of $C_{X / S}^{p}$ by

$$
D_{X / S}^{p}=\left(\mathcal{L}_{(X / G) / S}^{p} \rightarrow\left(\mathbb{R} \pi_{*} \Omega_{Y_{0} / S}^{p}\right)^{G} \rightarrow\left(\mathbb{R} \pi_{*} \Omega_{Y_{1} / S}^{p}\right)^{G} \rightarrow \cdots\right) .
$$

Now taking $G$-invariants commutes with $\mathbb{L} j_{s}^{*}$. Using (2.14c) and (2.14d), we have

$$
\mathbb{L} j_{s}^{*}\left(\left(\mathbb{R} \tilde{\pi}_{*} \Omega_{Y_{i} / S}^{p}\right)^{G}\right) \simeq\left(\mathbb{L} j_{s}^{*}\left(\mathbb{R} \tilde{\pi}_{*} \Omega_{Y_{i} / S}^{p}\right)\right)^{G} \simeq\left(\mathbb{R} q_{*} \Omega_{\left(Y_{i}\right)_{s}}^{p}\right)^{G} \simeq \mathbb{R} q_{*}\left(\underline{\Omega}_{\left.\left(Y_{i}\right)_{s} / G\right)}^{p}\right) .
$$

Finally, observe that a similar argument as that used to prove (2.3) shows that

$$
\underline{\Omega}_{X_{s} / G}^{p} \simeq\left(\mathbb{R} q_{*} \underline{\Omega}_{\left(\left(Y_{0}\right)_{s} / G\right)}^{p} \rightarrow \mathbb{R} q_{*} \underline{\Omega}_{\left(\left(Y_{1}\right)_{s} / G\right)}^{p} \rightarrow \cdots\right) .
$$

Indeed, $X_{s} / G, Y_{s} / G$, and the $\left(E_{i}\right)_{s} / G$ satisfy the same hypotheses as do $X, Y$, and the $E_{i}$, except for smoothness, so that the results in [7, 4.8, 4.10, 4.11] apply. It follows that

$$
j_{s}^{*}\left[D_{X / S}^{p}\right] \simeq\left[C_{\left(X_{s} / G\right)}^{p}\right] .
$$

Since the class of $\left[C_{X_{s} / G}^{p}\right]$ in $K_{0}(s)=\mathbb{Z}$ is $\chi^{p}\left(X_{s} / G\right)$, it is independent of $s$.

\section{ISOLATED (HYPER)SURFACE SINGULARITIES}

In this section we consider the du Bois invariants of a two-dimensional isolated hypersurface singularity $X$. That is, $X=\operatorname{Spec} R$ where $R=F[x, y, z] /(f(x, y, z))$ and $\Omega_{R / F}^{3} \cong R /\left(\frac{\partial f}{\partial x}, \frac{\partial f}{\partial y}, \frac{\partial f}{\partial z}\right)$ is supported at the origin (i.e., the unique singular point $x_{0}$ is defined by the maximal ideal $\left.(x, y, z)\right)$. The analytic analogues of some of our results are due to Steenbrink and may be found in Wahl's paper 21.

We will need the following well known calculation of $\Omega_{R}^{p}=\Omega_{R / F}^{p}$. Recall that the Tjurina number $\tau$ is:

$$
\tau=\text { length }_{R}\left(R /\left(\frac{\partial f}{\partial x}, \frac{\partial f}{\partial y}, \frac{\partial f}{\partial z}\right)\right) .
$$

Lemma 3.1. Let $X=\operatorname{Spec} R$ be a 2-dimensional isolated hypersurface singularity. Then each of the following $R$-modules has length equal to $\tau$ :

$$
\Omega_{R}^{3} \cong \operatorname{Ext}_{R}^{1}\left(\Omega_{R}^{1}, R\right) \cong \operatorname{Ext}_{R}^{2}\left(\Omega_{R}^{2}, R\right), \quad \operatorname{tors}\left(\Omega_{R}^{2}\right) \cong \operatorname{Ext}_{R}^{1}\left(\Omega_{R}^{2}, R\right) .
$$

Proof. Write $R=P / f$, where $P=F[x, y, z]$, and consider the complex $\mathcal{K}$ of free $R$-modules, whose boundary maps are induced by exterior multiplication with $d f$, indexed with $R$ in degree 0 :

$$
\mathcal{K}: \quad 0 \rightarrow R \stackrel{\wedge d f}{\rightarrow} \Omega_{P}^{1} \otimes_{P} R \stackrel{\wedge d f}{\rightarrow} \Omega_{P}^{2} \otimes_{P} R \stackrel{\wedge d f}{\rightarrow} \Omega_{P}^{3} \otimes_{P} R \rightarrow 0 .
$$

By [16, p. 326], the $n$-th cohomology of the complex $\mathcal{K}$ is the torsion submodule of $\Omega_{R}^{n}$. In the isolated singularity case considered here, it follows from Lebelt's results [12] (see also [15, Prop. 1]) that $\Omega_{R}^{n}$ is a torsionfree module for $n \leq 1$. In particular, we have the free resolutions:

$$
\begin{gathered}
0 \rightarrow R \stackrel{\wedge d f}{\rightarrow} \Omega_{P}^{1} \otimes_{P} R \rightarrow \Omega_{R}^{1} \rightarrow 0, \\
0 \rightarrow R \stackrel{\wedge d f}{\rightarrow} \Omega_{P}^{1} \otimes_{P} R \stackrel{\wedge d f}{\rightarrow} \Omega_{P}^{2} \otimes_{P} R \rightarrow \Omega_{R}^{2} \rightarrow 0 .
\end{gathered}
$$


Moreover the perfect pairing $\Omega_{P}^{p} \otimes_{P} \Omega_{P}^{3-p} \rightarrow \Omega_{P}^{3} \cong P$ induces a perfect pairing $\mathcal{K}^{p} \otimes_{R} \mathcal{K}^{3-p} \rightarrow \mathcal{K}^{3} \cong R$. From this we get an isomorphism of complexes $\operatorname{Hom}_{R}(\mathcal{K}, R)[-3] \cong \mathcal{K}$. It follows that

$$
\begin{gathered}
\operatorname{Ext}_{R}^{1}\left(\Omega_{R}^{1}, R\right)=H^{3}(\mathcal{K})=\Omega_{R}^{3} \cong R /\left(\frac{\partial f}{\partial x}, \frac{\partial f}{\partial y}, \frac{\partial f}{\partial z}\right), \\
\operatorname{Ext}_{R}^{1}\left(\Omega_{R}^{2}, R\right)=H^{2}(\mathcal{K})=\operatorname{tors} \Omega_{R}^{2} .
\end{gathered}
$$

By definition, the length of the first of these modules is $\tau$; by [15, Thm. 3], the second module also has length $\tau$.

Recall the definition of the (generalized) du Bois invariants $b^{p, q}$ from (2.8).

Proposition 3.2. Let $X=\operatorname{Spec} R$ be a 2-dimensional isolated hypersurface singularity. Then the following hold:

(a) $b^{p, q}=0$ unless $p+q \in\{1,2\}$.

(b) $b^{1-q, q}=b^{2-q, q}=\tau$ for all $q<0$.

(c) $b^{0,2}=0$ and $b^{0,1}=-\chi^{0}$.

Proof. To prove (a), note that for $q>0$, it is a particular case of a general statement for isolated singularities proved by Steenbrink in [18, Thm. 1], since $b^{p, q}$ is the length of $\mathbb{H}^{q}\left(X, \underline{\Omega}_{X}^{p}\right)$ by (2.7). In our case, Steenbrink's result is immediate from GrauertRiemenschneider vanishing [9, Satz 2.3] and from the fact, proved in [6. Prop. 2.6], that for an affine surface $X$,

$$
H_{\mathrm{cdh}}^{2}\left(X, \Omega^{p}\right)=0 \quad(p \geq 0) .
$$

If $q=0$ and $p>2$, (a) holds since then $a^{*} \Omega^{p}=0$. If $q=p=0$, it holds since $R$ is normal, hence seminormal. For $q=-1$, (a) holds because $\Omega_{R}^{p}=0$ for $p>3$ and $R$ and $\Omega_{R}^{1}$ are torsionfree; see [5, Lemma 5.6 and Remark 5.6.1]. For $q \leq-2$, we have

$$
H_{q}\left(C_{X}^{p}\right)=D_{-1-q}^{(p)}(R)=H H_{p-q-1}^{(p)}(R)=\operatorname{tors}\left(\Omega_{R}^{p+q+1}\right),
$$

which is zero unless $p+q \in\{1,2\}$, by a result of Michler [16].

Assertion (b) follows from (3.4) and the fact that the kernel of $\Omega_{R}^{n} \rightarrow H_{\mathrm{cdh}}^{0}\left(X, \Omega^{n}\right)$ is $\operatorname{tors}\left(\Omega_{R}^{n}\right)$ (see [5, Lemma 5.6 and Remark 5.6.1]), using [15, Thm. 3] (see Lemma 3.1).

For assertion (c), the vanishing of $b^{0,2}$ is a particular case of (3.3). The other assertion follows from part (a) and the definition (see 2.9) of $\chi^{0}$.

Proposition 3.5. Let $X=\operatorname{Spec} R$ be a 2-dimensional isolated hypersurface singularity. Further let $\pi: Y \rightarrow X$ be a good resolution, $E$ the exceptional divisor, $E_{1}, \ldots, E_{r}$ its reduced irreducible components, $g_{i}$ the genus of $E_{i}$, and $l$ the number of loops in the incidence graph. Put $g=\sum_{i} g_{i}$ and $p_{g}=\operatorname{length}_{R} H^{1}\left(Y, \mathcal{O}_{Y}\right)$.

(a) The map $H_{\mathrm{cdh}}^{n}(X, \mathcal{O}) \rightarrow H_{\mathrm{cdh}}^{n}(Y, \mathcal{O})=H^{n}(Y, \mathcal{O})$ is an isomorphism for $n \neq 1$, and an injection for $n=1$. We have

$$
b^{0,1}=p_{g}-g-l .
$$

In particular, $H_{\mathrm{cdh}}^{1}(X, \mathcal{O}) \rightarrow H^{1}(Y, \mathcal{O})$ is an isomorphism $\Longleftrightarrow g=l=0$.

(b) $H_{\mathrm{cdh}}^{n}\left(X, \Omega^{2}\right) \cong H^{n}\left(Y, \Omega^{2}\right)$ for $n \geq 0$. In particular, $H_{\mathrm{cdh}}^{n}\left(X, \Omega^{2}\right)=0$ for $n \geq 1$.

(c) $\operatorname{Ext}_{R}^{i}\left(H^{0}\left(Y, \Omega^{2}\right), R\right) \cong H^{i}\left(Y, \mathcal{O}_{Y}\right)$. In particular, $\operatorname{Ext}_{R}^{2}\left(H^{0}\left(Y, \Omega^{2}\right), R\right)=0$.

(d) $b^{1,0} \leq \tau$.

(e) $b^{2,0}=\tau-p_{g}$ and $\chi^{2}=-p_{g}$. 
Proof. To prove (a), observe that $R$ is normal and $Y \rightarrow X$ is projective, so that $R=H_{\mathrm{cdh}}^{0}(X, \mathcal{O})=H^{0}(Y, \mathcal{O})$ by Zariski's Main Theorem (and [5, Proposition 2.5]). Since $Y \rightarrow X$ has fibers of dimension at most 1 and $X$ is affine,

$$
H^{2}(Y, \mathcal{F})=H^{0}\left(X, \mathbb{R}^{2} \pi_{*} \mathcal{F}\right)=0
$$

for all coherent sheaves $\mathcal{F}$. In particular, $H^{2}(Y, \mathcal{O})=0$. Similarly, $H_{\text {cdh }}^{2}(X, \mathcal{O})=0$ by [3, Theorem 6.1]. Since Sing $X=\left\{x_{0}\right\}$, we have a blow-up square

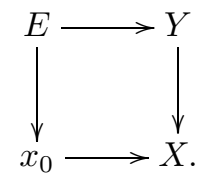

From the Mayer-Vietoris sequence associated to this square, we extract the short exact sequence

$$
0 \rightarrow H_{\mathrm{cdh}}^{1}(X, \mathcal{O}) \rightarrow H^{1}(Y, \mathcal{O}) \rightarrow H_{\mathrm{cdh}}^{1}(E, \mathcal{O}) \rightarrow 0 .
$$

Hence $b^{0,1}=\operatorname{length}_{R} H^{1}(Y, \mathcal{O})-\operatorname{length}_{R} H_{\text {cdh }}^{1}(E, \mathcal{O})$. Applying descent to the cover $\coprod_{i} E_{i} \rightarrow E$, we obtain length $H_{\mathrm{cdh}}^{1}(E, \mathcal{O})=l+g$.

For (b), the isomorphisms $H_{\mathrm{cdh}}^{n}\left(X, \Omega^{2}\right) \cong H^{n}\left(Y, \Omega^{2}\right)$ follow from the MayerVietoris sequence associated to the square (3.7). By Grauert-Riemenschneider vanishing [9, Satz 2.3], $\mathbb{R} \pi_{*} \Omega_{Y}^{2} \simeq \pi_{*} \Omega_{Y}^{2}$, so $H^{n}\left(Y, \Omega^{2}\right)=H^{0}\left(X, R^{n} \pi_{*} \Omega_{Y}^{2}\right)$ vanishes for $n>0$ because $X$ is affine.

To prove (c), recall that $\omega_{X} \cong \mathcal{O}_{X}[2]$ because $X$ is an affine hypersurface. For any bounded complex of quasi-coherent sheaves $\mathcal{F}^{\bullet}$ on $Y$, Grothendieck-Serre duality gives a quasi-isomorphism:

$$
\mathbb{R} \pi_{*} \mathbb{R} \operatorname{Hom}_{Y}\left(\mathcal{F}^{\bullet}, \Omega_{Y}^{2}\right) \simeq \mathbb{R} \operatorname{Hom}_{X}\left(\mathbb{R} \pi_{*} \mathcal{F}^{\bullet}, \mathcal{O}_{X}\right) .
$$

Taking $\mathcal{F}^{\bullet}=\Omega_{Y}^{p}$ and using the duality pairing on $Y$,

$$
\mathbb{R} \operatorname{Hom}_{Y}\left(\Omega_{Y}^{p}, \Omega_{Y}^{2}\right) \simeq \operatorname{Hom}_{Y}\left(\Omega_{Y}^{p}, \Omega_{Y}^{2}\right) \cong \Omega_{Y}^{2-p},
$$

we get a spectral sequence

$$
\operatorname{Ext}_{R}^{i}\left(H^{j}\left(Y, \Omega^{p}\right), R\right) \Rightarrow H^{i-j}\left(Y, \Omega^{2-p}\right) .
$$

Taking $p=2$ and using Grauert-Riemenschneider vanishing [9, Satz 2.3], which gives $H^{j}\left(Y, \Omega^{2}\right)=0$ for $j>0$, we obtain the conclusion of (c):

$$
\operatorname{Ext}_{R}^{i}\left(H^{0}\left(Y, \Omega^{2}\right), R\right) \cong H^{i}\left(Y, \mathcal{O}_{Y}\right) .
$$

In particular, by (3.6), $\operatorname{Ext}_{R}^{2}\left(H^{0}\left(Y, \Omega^{2}\right), R\right)=0$.

To prove (d), recall that $b^{1,0}$ is the length of the $R$-module $L=\mathbb{H}^{0}\left(C_{X}^{1}\right)$. Since $b^{1,-1}=0$ by Proposition 3.2, it follows from (2.7) that we have an exact sequence

$$
0 \rightarrow \Omega_{R}^{1} \rightarrow H_{\mathrm{cdh}}^{0}\left(X, \Omega^{1}\right) \rightarrow L \rightarrow 0 .
$$

From (3.9) we get the exact sequence

$$
\operatorname{Ext}_{R}^{1}\left(\Omega_{R}^{1}, R\right) \rightarrow \operatorname{Ext}_{R}^{2}(L, R) \rightarrow \operatorname{Ext}_{R}^{2}\left(H_{\mathrm{cdh}}^{0}\left(X, \Omega^{1}\right), R\right) .
$$

From the spectral sequence (3.8) with $p=1$, we have an exact sequence

$$
\operatorname{Hom}_{R}\left(H^{1}\left(Y, \Omega^{1}\right), R\right) \stackrel{d_{2}}{\longrightarrow} \operatorname{Ext}_{R}^{2}\left(H^{0}\left(Y, \Omega^{1}\right), R\right) \rightarrow H^{2}\left(Y, \Omega^{1}\right) .
$$

Since the $R$-module $H^{1}\left(Y, \Omega^{1}\right)$ is supported at $x_{0}, \operatorname{Hom}_{R}\left(H^{1}\left(Y, \Omega^{1}\right), R\right)=0$. The right side also vanishes, by (3.6), so we get $\operatorname{Ext}_{R}^{2}\left(H^{0}\left(Y, \Omega^{1}\right), R\right)=0$. 
By part (a), the map $H_{\text {cdh }}^{0}\left(X, \Omega^{1}\right) \rightarrow H^{0}\left(Y, \Omega^{1}\right)$ is injective, so the map

$$
\operatorname{Ext}_{R}^{2}\left(H^{0}\left(Y, \Omega^{1}\right), R\right) \rightarrow \operatorname{Ext}_{R}^{2}\left(H_{\text {cdh }}^{0}\left(X, \Omega^{1}\right), R\right)
$$

is surjective and hence

$$
\operatorname{Ext}_{R}^{2}\left(H_{\mathrm{cdh}}^{0}\left(X, \Omega^{1}\right), R\right)=0 .
$$

From (3.10) we get that $\operatorname{Ext}_{R}^{1}\left(\Omega_{R}^{1}, R\right) \rightarrow \operatorname{Ext}_{R}^{2}(L, R)$ is surjective and hence

$$
\begin{aligned}
b^{1,0}=\operatorname{length}_{R}(L) & =\operatorname{length}_{R}\left(\operatorname{Ext}_{R}^{2}(L, R)\right) \\
& \leq \operatorname{length}_{R}\left(\operatorname{Ext}_{R}^{1}\left(\Omega_{R}^{1}, R\right)\right) \\
& =\tau, \text { by Lemma 3.1. }
\end{aligned}
$$

To prove (e), define finite length $R$-modules $N$ and $M$ so that

$$
0 \rightarrow N \rightarrow \Omega_{R}^{2} \rightarrow H^{0}\left(Y, \Omega^{2}\right) \rightarrow M \rightarrow 0
$$

is exact. By definition (2.8) and the fact that $R$ is Gorenstein, we get

$$
b^{2,0}=\operatorname{length}_{R}(M)=\operatorname{length}_{R}\left(\operatorname{Ext}_{R}^{2}(M, R)\right) .
$$

Because $N$ has finite length, $\operatorname{Ext}^{i}(N, R)=0$ for $i<2$, and hence there are isomorphisms

$$
\operatorname{Ext}^{i}\left(\Omega_{R}^{2} / N, R\right) \stackrel{\widetilde{\sim}}{\rightarrow} \operatorname{Ext}^{i}\left(\Omega_{R}^{2}, R\right) \quad(i<2) .
$$

Using this together with part (c) and (3.11), we get an exact sequence

$$
0 \rightarrow H^{1}\left(Y, \mathcal{O}_{Y}\right) \rightarrow \operatorname{Ext}_{R}^{1}\left(\Omega_{R}^{2}, R\right) \rightarrow \operatorname{Ext}_{R}^{2}(M, R) \rightarrow 0 .
$$

Using this sequence, and taking into account Lemma 3.1 and (3.12), we get

$$
b^{2,0}=\tau-\operatorname{length}_{R} H^{1}(Y, \mathcal{O})=\tau-p_{g} .
$$

By Proposition 3.2(a,b), this yields $\chi^{2}=b^{2,0}-\tau=-p_{g}$.

\section{WAHL'S EXAMPLE}

Using the general results of the preceding sections, we can now prove:

Theorem 4.1. Let $F$ be a field of characteristic 0 and

$$
R=F[x, y, z] /\left(z^{2}+y^{3}+x^{10}+a x^{7} y\right),
$$

for any nonzero $a \in F$. Then $b^{0,1}=1$ and $b^{1,1}=0$. That is,

(a) $H_{\text {cdh }}^{1}(R, \mathcal{O}) \cong F$ and

(b) $H_{\mathrm{cdh}}^{1}\left(R, \Omega_{/ F}^{1}\right)=0$.

In particular, if $F$ is an algebraic field extension of $\mathbb{Q}$, then $R$ gives a negative answer to Bass' question for $n=0$ :

$$
K_{0}(R)=K_{0}(R[t]) \text { but } K_{0}\left(R\left[t_{1}, t_{2}\right]\right) \cong K_{0}(R) \oplus s t F[s, t] .
$$

Remark 4.1.1. The cdh cohomology groups in question may also be computed using an explicit description of a resolution of singularities, together with the selfintersection numbers of the exceptional components. For the surface in Theorem 4.1 for all values of $a$ (including 0), the resolution data was checked for us by Liz Sell and is displayed in Figure 1. 


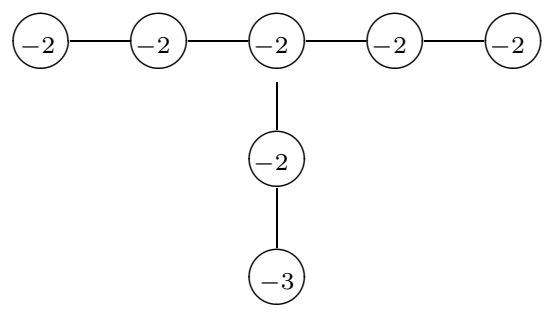

Figure 1. The resolution graph for $z^{2}+y^{3}+x^{10}+a x^{7} y$

The proof we shall give here will be a straightforward application of the invariance of $\chi^{p}$ (Theorem 2.14), applied to the specific example:

$$
X=\operatorname{Spec} F[x, y, z, t] /\left(z^{2}+y^{3}+x^{10}+t x^{7} y\right) .
$$

Consider the map $X \rightarrow S=$ Spec $F[t]$ induced by the obvious inclusion of rings, and write $X_{s}$ for the fiber over $s \in S$. When $s$ is the point $t=a$ we have $X_{s}=\operatorname{Spec}(R)$ for the ring $R$ in Theorem 4.1 ,

Proposition 4.3. Let $X$ be the affine variety of (4.2). Then the integer $\chi^{p}\left(X_{s}\right)$ is independent of the choice of closed point $s \in S$.

Proof. Since the value of $\chi^{p}$ does not change upon passing to a finite extension, we may assume that $F$ contains a primitive 30 -th root of unity. Put

$$
\tilde{X}=\operatorname{Spec} F[u, v, w, t] /\left(u^{30}+v^{30}+w^{30}+t u^{21} v^{10}\right) .
$$

Let $G=\mu_{3} \times \mu_{10} \times \mu_{15}$ act on $\tilde{X}$ by scalar multiplication on the variables $x, y, z$ so that the assignment $x=u^{3}, y=v^{10}$ and $z=w^{15}$ identifies $X$ with $\tilde{X} / G$.

The map $X \rightarrow S$ is a flat local complete intersection whose singular locus is defined by $x=y=z=0$ and hence maps isomorphically onto $S$. The singular locus of $\tilde{X}$ is defined by $u=v=w=0$ and hence also maps isomorphically onto $S$. Let $\tilde{Y}$ be the blowup of $\tilde{X}$ along its singular locus. Then

$$
\tilde{Y}=\operatorname{Proj}\left(\frac{F[t, u, v, w, A, B, C]}{\left(A^{30}+B^{30}+C^{30}+t u B^{10} A^{20}, u B-v A, u C-w A, v C-w B\right)}\right),
$$

where $t, u, v, w$ have degree 0 and $A, B, C$ have degree 1 . Direct calculations show that $\tilde{Y} \rightarrow S$ is smooth and the fiber of $\tilde{Y} \rightarrow \tilde{X}$ over $\tilde{X}_{\text {sing }}$ is

$$
\tilde{E}=\operatorname{Proj} F[t, A, B, C] /\left(A^{30}+B^{30}+C^{30}\right) \cong S \times E_{0},
$$

where $E_{0}$ is a smooth curve. We see that all the hypotheses of Theorem 2.14 are satisfied.

Since $X_{0}$ is quasi-homogeneous, its du Bois invariants may be computed, as shown in the following example. These calculations and the above results lead to the proof below of Theorem 4.1 .

Example 4.4. The surface $X_{0}=\operatorname{Spec} F[x, y, z] /\left(z^{2}+y^{3}+x^{10}\right)$ is discussed by Wahl in [21, 4.4]. Elementary calculations, described in [21, 4.3], give that $\tau=1 \cdot 2 \cdot 9=18$, $g=0$ and

$$
p_{g}=\operatorname{dim}\left(F[x, y, z] /\left(\frac{\partial f}{\partial x}, \frac{\partial f}{\partial y}, \frac{\partial f}{\partial z}\right)\right)_{\leq 2}=1,
$$

where $f=z^{2}+y^{3}+x^{10}$. Moreover, as with any isolated normal surface singularity defined by a nonnegatively graded ring, we have $l=0$ by [17, Theorem 2.3.1]. 
(Or, one may see that $l=0$ from the graph of Figure 1.) Using Lemma 2.10 and Proposition 3.5 (a,e), this yields

$$
b^{1,1}=b^{0,1}=p_{g}-g-l=1, \quad b^{1,0}=b^{2,0}=\tau-p_{g}=17 .
$$

By Proposition 3.2 (a), $\chi^{0}=-b^{0,1}=-1, \chi^{1}=b^{1,0}-b^{1,1}=16, \chi^{2}=-1$.

Proof of Theorem 4.1. By Theorem 2.14, $\chi^{p}\left(X_{s}\right)$ does not depend on $s$, and we write $\chi^{p}=\chi_{s}^{p}$. By Proposition $3.2(\mathrm{c}), b^{0,1}=-\chi^{0}$ is also independent of $s$. For the choice $s=0$, we have $b_{0}^{0,1}=1$ by [21, 4.4] (see Example 4.4). This proves assertion (a). To compute $b^{1,1}$ when $a \neq 0$, we use the calculation of $\tau\left(X_{a}\right)$ given in [21, 4.4]:

$$
\tau\left(X_{a}\right)= \begin{cases}18 & a=0 \\ 16 & a \neq 0\end{cases}
$$

By Proposition 3.5 (d),

$$
b^{1,0}\left(X_{a}\right) \leq \tau\left(X_{a}\right)=16 \text { for all } a \neq 0 .
$$

By the invariance of $\chi^{1}$ (see Proposition 4.3), Example 4.4 and (4.6), we have

$$
\begin{aligned}
16=\chi^{1} & =b^{1,0}\left(X_{a}\right)-b^{1,1}\left(X_{a}\right) \\
& \leq 16-b^{1,1}\left(X_{a}\right)
\end{aligned}
$$

for any $a \neq 0$, and hence $0=b^{1,1}\left(X_{a}\right)=\operatorname{dim}_{F} H_{\text {cdh }}^{1}\left(X_{a}, \Omega^{1}\right)$.

The final assertion follows from Theorem 1.1.

Remark 4.7. We conclude with a few remarks.

(a) In (4.5) of the proof, we refer to the calculation of the Tjurina numbers $\tau$ stated by Wahl in [21, 4.4]. These can be checked directly using the Tjurina function of the Singular library sing.lib ([11], [10]).

(b) Steenbrink uses analytic methods to define an invariant $\alpha$ and proves that $b^{1,1}=p_{g}-g-l-\alpha ;$ see [21, (1.9.1)]. Comparing with Proposition 3.2(a), and using GAGA, we see that $\alpha=b^{0,1}-b^{1,1}$. It is this invariant that is computed by Wahl in [21, 4.4].

(c) If $R_{F}=F[x, y, z] /\left(z^{2}+y^{3}+x^{10}\right)$ and $F$ is not algebraic over $\mathbb{Q}$, then $N K_{0}\left(R_{F}\right)$ is nonzero. Indeed, $N K_{0}\left(R_{F}\right) \cong \Omega_{F / \mathbb{Q}}^{1} \otimes_{F} t F[t]$. This follows from [5. (7.4)], which says that

$$
N K_{0}\left(R_{F}\right) \cong N K_{0}\left(R_{\mathbb{Q}}\right) \otimes_{\mathbb{Q}} F \oplus N K_{-1}\left(R_{\mathbb{Q}}\right) \otimes_{\mathbb{Q}} \Omega_{F / \mathbb{Q}}^{1},
$$

since $N K_{0}\left(R_{\mathbb{Q}}\right)=0$ and $N K_{-1}\left(R_{F}\right) \cong t F[t]$ by Theorems 1.1(b) and 4.1(a).

\section{ACKNOWLEDGEMENTS}

The authors would like to thank M. Schlichting, whose contributions go beyond the collaboration [3]. We would also like to thank W. Vasconcelos and L. Avramov for useful discussions, and E. Sell and J. Wahl for their help in checking our resolution of singularities. 


\section{REFERENCES}

1. Théorie des intersections et théorème de Riemann-Roch, Lecture Notes in Mathematics, Vol. 225, Springer-Verlag, Berlin, 1971, Séminaire de Géométrie Algébrique du Bois-Marie 1966-1967 (SGA 6), Dirigé par P. Berthelot, A. Grothendieck et L. Illusie. Avec la collaboration de D. Ferrand, J. P. Jouanolou, O. Jussila, S. Kleiman, M. Raynaud et J. P. Serre. MR0354655(50:7133)

2. Hyman Bass, Some problems in "classical" algebraic K-theory, Algebraic K-theory, II: "Classical" algebraic $K$-theory and connections with arithmetic (Proc. Conf., Battelle Memorial Inst., Seattle, Wash., 1972), Springer, Berlin, 1973, pp. 3-73. Lecture Notes in Math., Vol. 342. MR0409606 (53:13358)

3. G. Cortiñas, C. Haesemeyer, M. Schlichting, and C. Weibel, Cyclic homology, cdh-cohomology and negative K-theory, Ann. of Math. (2) 167 (2008), no. 2, 549-573. MR 2415380 (2009c:19006)

4. G. Cortiñas, C. Haesemeyer, Mark E. Walker, and C. Weibel, The K-theory of toric varieties, Trans. Amer. Math. Soc. 361 (2009), no. 6, 3325-3341. MR 2485429 (2010b:19001)

5. , Bass' NK groups and cdh-fibrant Hochschild homology, preprint. Inventiones Math. 181 (2010), 421-448.

6. G. Cortiñas, C. Haesemeyer, and C. Weibel, K-regularity, cdh-fibrant Hochschild homology, and a conjecture of Vorst, J. Amer. Math. Soc. 21 (2008), no. 2, 547-561. MR2373359 (2008k:19002)

7. Philippe Du Bois, Complexe de de Rham filtré d'une variété singulière, Bull. Soc. Math. France 109 (1981), no. 1, 41-81. MR613848 (82j:14006)

8. B. L. Fĕ̌gin and B. L. Tsygan, Additive K-theory and crystalline cohomology, Funktsional. Anal. i Prilozhen. 19 (1985), no. 2, 52-62, 96. MR800920 (88e:18008)

9. Hans Grauert and Oswald Riemenschneider, Verschwindungssätze für analytische Kohomologiegruppen auf komplexen Räumen, Invent. Math. 11 (1970), 263-292. MR0302938 (46:2081)

10. G.-M. Greuel and G. Pfister, A singular 3.0 library for invariants of singularities, 2005, sing.lib.

11. G.-M. Greuel, G. Pfister, and H. Schönemann, Singular 3.0 - A computer algebra system for polynomial computations, 2005, http://www.singular.uni-kl.de.

12. Karsten Lebelt, Torsion äußerer Potenzen von Moduln der homologischen Dimension 1, Math. Ann. 211 (1974), 183-197. MR0371875 (51:8092)

13. Ben Lee, Local acyclic fibrations and the de Rham complex, Homology, Homotopy Appl. 11 (2009), no. 1, 115-140. MR2506129

14. Jean-Louis Loday, Cyclic homology, Grundlehren der Mathematischen Wissenschaften [Fundamental Principles of Mathematical Sciences], vol. 301, Springer-Verlag, Berlin, 1992; Appendix E by María O. Ronco. MR1217970 (94a:19004)

15. Ruth Michler, Torsion of differentials of hypersurfaces with isolated singularities, J. Pure Appl. Algebra 104 (1995), no. 1, 81-88. MR.1359692 (96k:13036)

16. Ruth Ingrid Michler, Hodge-components of cyclic homology for affine quasi-homogeneous hypersurfaces, Astérisque (1994), no. 226, 10, 321-333, K-theory (Strasbourg, 1992). MR.1317123 (96a:19003)

17. Peter Orlik and Philip Wagreich, Isolated singularities of algebraic surfaces with $\mathbb{C}^{*}$ action, Ann. of Math. (2) 93 (1971), 205-228. MR0284435 (44:1662)

18. Joseph H. M. Steenbrink, Du Bois invariants of isolated complete intersection singularities, Ann. Inst. Fourier (Grenoble) 47 (1997), no. 5, 1367-1377. MR1600383 (99f:32058)

19. Andrei Suslin and Vladimir Voevodsky, Bloch-Kato conjecture and motivic cohomology with finite coefficients, The arithmetic and geometry of algebraic cycles (Banff, AB, 1998), NATO Sci. Ser. C Math. Phys. Sci., vol. 548, Kluwer Acad. Publ., Dordrecht, 2000, pp. 117-189. MR:1744945 (2001g:14031)

20. Carlo Traverso, Seminormality and Picard group, Ann. Scuola Norm. Sup. Pisa (3) 24 (1970), 585-595. MR0277542 (43:3275)

21. Jonathan M. Wahl, A characterization of quasihomogeneous Gorenstein surface singularities, Compositio Math. 55 (1985), no. 3, 269-288. MR799816 (87e:32013)

22. Charles A. Weibel, An introduction to homological algebra, Cambridge Studies in Advanced Mathematics, vol. 38, Cambridge University Press, Cambridge, 1994. MR1269324 (95f:18001) 
Departamento Matemática, FCEyN-Universidad de Buenos Aires, Ciudad Universitaria Pab 1, 1428 Buenos Aires, Argentina

E-mail address: gcorti@dm.uba.ar

Department of Mathematics, University of California, Los Angeles, California 90095

E-mail address: chh@math.ucla.edu

Department of Mathematics, University of Nebraska, Lincoln, Lincoln, Nebraska 68588

E-mail address: mwalker5@math.unl.edu

Department of Mathematics, Rutgers University, New Brunswick, New Jersey 08901

E-mail address: weibel@math.rutgers.edu 